\title{
Changes of volatiles' attribute in durian pulp during freeze- and spray-drying process.
}

\begin{abstract}
Changes of volatile profile from durian (Durio zibethinus) pulp during freeze-drying and spray-drying process were studied using headspace SPME coupled to fast GC-TOFMS. Results showed that 4 esters diminished in the volatiles' composition of freeze-dried pulp and spray drying caused losses of 14 volatile constituents from the profile. Formation of new volatiles was induced in spray-dried durian powder, comprising 5 aldehydes, 1 ketone, 1 furan and 1 pyrrole compounds. Furthermore, dramatic decline was observed during freeze drying for the amount of major durian aroma included propanethiol, ethyl propanoate, propyl propanoate, ethyl 2-methylbutanoate and diethyl disulfide, ranging from $71 \%$ to $97 \%$ while $98 \%$ to $99 \%$ amount of such volatiles were vanished during spray-drying process.
\end{abstract}

Keyword: Durian; Spray drying; Freeze drying; SPME; Fast GC; Time-of-Flight Mass Spectrometry. 